Article

\title{
CLE-CLAVATA1 Signaling Pathway Modulates Lateral Root Development under Sulfur Deficiency
}

\author{
Wei Dong, Yinghua Wang and Hideki Takahashi *(D) \\ Department of Biochemistry and Molecular Biology, Michigan State University, East Lansing, MI 48824, USA; \\ wdong@msu.edu (W.D.); wangyi64@msu.edu (Y.W.) \\ * Correspondence: htakaha@msu.edu
}

Received: 2 March 2019; Accepted: 17 April 2019; Published: 18 April 2019

\begin{abstract}
Plant root system architecture changes drastically in response to availability of macronutrients in the soil environment. Despite the importance of root sulfur (S) uptake in plant growth and reproduction, molecular mechanisms underlying root development in response to $S$ availability have not been fully characterized. We report here on the signaling module composed of the CLAVATA3 (CLV3)/EMBRYO SURROUNDING REGION (CLE) peptide and CLAVATA1 (CLV1) leucine-rich repeat receptor kinase, which regulate lateral root (LR) development in Arabidopsis thaliana upon changes in $\mathrm{S}$ availability. The wild-type seedlings exposed to prolonged $\mathrm{S}$ deficiency showed a phenotype with low LR density, which was restored upon sulfate supply. In contrast, the $c l v 1$ mutant showed a higher daily increase rate of LR density relative to the wild-type under prolonged S deficiency, which was diminished to the wild-type level upon sulfate supply, suggesting that CLV1 directs a signal to inhibit LR development under S-deficient conditions. CLE2 and CLE3 transcript levels decreased under S deficiency and through CLV1-mediated feedback regulations, suggesting the levels of CLE peptide signals are adjusted during the course of LR development. This study demonstrates a fine-tuned mechanism for LR development coordinately regulated by CLE-CLV1 signaling and in response to changes in S availability.
\end{abstract}

Keywords: Arabidopsis thaliana; CLE peptide; CLAVATA1; root system architecture; small signaling peptide; sulfate; sulfur

\section{Introduction}

Plant roots optimize nutrient uptake capacity by altering the root system architecture (RSA) in the soil environment [1]. Changes in nutrient availabilities have a distinct effect on RSA depending on the nutrient types and the amount supplied or locally concentrated in the soil environment [2]. The macronutrient sulfur (S), in the form of sulfate, is a mobile resource found in deeper soil profiles [1]. It is proposed that a combination of a thick and deep primary root (PR) with few and long lateral roots (LRs) can improve the uptake of S [3]. To achieve the adjustment of RSA, individual root traits can be regulated independently in response to changes in nutrient availabilities and patterns of nutrient distributions in the soil environment [4]. Among all root traits, LRs are phenotypically evaluated by length, total numbers, and density, which is considered a major determinant of RSA [5]. Changes in sulfate availability have a variable effect on LR development. For example, several studies have demonstrated that $\mathrm{S}$ deficiency leads to reduction in LR length [2,6-9] and LR number or density $[2,7,9]$. However, active growth of LR appears to be another response to sulfate starvation as it is described with longer LR length $[10,11]$ and higher LR number or density [11-13].

Studies on functional characterization of small signaling peptides (SSPs) reveal that several distinct groups of SSPs play important roles in plant root development [14-24]. Nitrogen (N)-responsive C-TERMINALLY ENCODED PEPTIDEs (CEPs) are a group of SSPs known to be functional as negative 
regulators of LR development under N-limited conditions [14-17], while they have also been shown to be involved in long-distance regulation of $\mathrm{N}$ uptake [18]. A few distinct members of the CLAVATA3 (CLV3)/EMBRYO SURROUNDING REGION (CLE) family are also well characterized as SSPs regulating LR development in Arabidopsis thaliana (A. thaliana) [19,24]. CLE1, -3, -4, and -7 are characterized particularly in relation to $\mathrm{N}$ nutritional responses as they are expressed in roots under $\mathrm{N}$ deficient conditions [19]. The LR phenotypes depicted in our previous study therefore highlight the CLE3 gene expression enhanced in roots as a potential mechanism suppressing LR development under low $\mathrm{N}$ supply or in response to systemic $\mathrm{N}$ demand signals [19]. CLE2 and CLE3 also demonstrate positive responses of gene expression after $\mathrm{N}$ resupply to $\mathrm{N}$-starved seedlings, as their transcript levels are shown to dramatically increase in response to nitrate and ammonium, respectively $[19,25]$. CLE1, $-2,-3,-4$, and -7 are predominantly expressed in pericycle cells in roots, while CLE1 and CLE5 promoters are also found to be active in epidermal cells of the primary root tip [19]. These partially overlapping expression patterns, environmental responses and feedback regulation suggest functional redundancy of these N-responsive CLE genes in LR development. In addition to mechanisms characterized in relation to the $\mathrm{N}$ status, CLE genes are also known to be transcriptionally modulated by changes in availability of other macronutrients including $S$, phosphorus $(\mathrm{P})$ and potassium $(\mathrm{K})[24,26]$, as well as perturbation of cellular status caused by phytohormones and environmental stimuli [24,27]. More specifically to responses to $S$ in roots, CLE12 and CLE2 are known to be up- and down-regulated, respectively, by $S$ deprivation [24]. Thus, CLE2 seems to be controlled by the S-responsive pathways in addition to being up-regulated by resupply of $\mathrm{N}$ [19]. In contrast, the S-responsive regulation of CLE3 gene expression has not been studied despite its roles in LR development documented in relation to responses to $\mathrm{N}$ nutrition. CLE2 peptide has been shown to physically bind to the CLE receptor CLAVATA1 (CLV1) [28], and CLE3 requires CLV1 to transmit signals to modulate LR development [19]. Based on these aspects of nutrient-responsive regulation of CLE2 and CLE3 gene expression and their specific relationship with CLV1, we focused on investigating the effect of $S$ on the CLE-CLV1 signaling pathway and LR development.

Here, we report the CLE-CLV1 signaling pathway is associated with S-responsive mechanisms modulating LR development in A. thaliana. The results shown in this study suggest a link between the morphological responses of LRs and the CLE-CLV1 signaling pathway, as well as S-responsive regulations of CLE2 and CLE3 genes in A. thaliana seedlings exposed to prolonged S deficiency.

\section{Results}

\subsection{CLAVATA1 Controls Lateral Root Development under S Deficiency}

To investigate the effect of $S$ supply on root development, the wild-type $A$. thaliana (accession Columbia-0 (Col-0)) were germinated and precultured on a $-\mathrm{S}(15 \mu \mathrm{M}$ sulfate) or $+\mathrm{S}(1500 \mu \mathrm{M}$ sulfate) medium for 7 days. The seedlings were then transferred to the medium with the same concentration of sulfate, or from the $-S$ preculture to the $+S$ medium, or from the $+S$ preculture to the $-S$ medium, and grown for 3 days to validate the effect of $S$ starvation and $S$ replenishment (Figure 1). The most significant changes in root morphology at Day 10 were the decrease in length and number of LRs after long-term limitation of $S$ (Figure 1a; plants transferred from $-S$ to $-S$ ) compared to the recovery of the roots observed in response to supply of sulfate (Figure $1 b$; plants transferred from $-S$ to $+S$ ). The PR growth was slightly enhanced when the seedlings were transferred from the $-S$ preculture to the $+S$ medium (Figure $1 \mathrm{a}, \mathrm{b}$ ). In contrast, the seedlings from the $+S$ preculture medium transferred either to the $+S$ or $-S$ medium showed no significant changes in the root morphological phenotypes (Figure 1c,d).

Based on these observations, we hypothesized the CLE-CLV1 signaling pathway, which has been shown to regulate LR development under N-starved conditions [19], would be involved in pathways modulating root growth under S-limited conditions. To test this hypothesis, we focused on studying the effect of $c l v 1$ mutations on root growth under conditions where the differences in root morphology were most significant. As mentioned, the most significant morphological changes were observed for 
the $-S$-precultured seedlings transferred to either the $-S$ or $+S$ medium (Figure 1a,b). The effect of $S$ supply on changes in root morphology was recorded at Day 7 before the transfer and during the 3 consecutive days in the post-transfer growth period until Day 10 (Figures 2-4). The results indicated that changes in S conditions had little influence on primary root growth (Figure 2). The clv1-101 mutant had slightly shorter PR values compared to its background wild-type accession (Col-0), regardless of changes in S conditions; however, the same phenotype was not observed in the clv1-4 mutant in comparison with its background wild-type accession Landsberg erecta (Ler) (Figure 2).

(a)

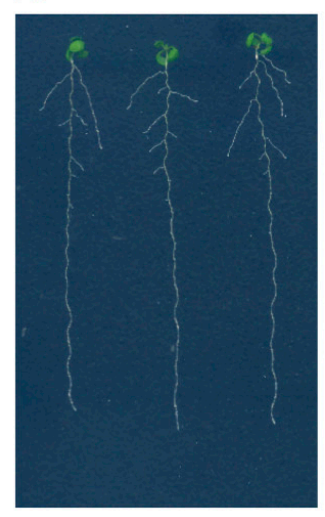

(b)

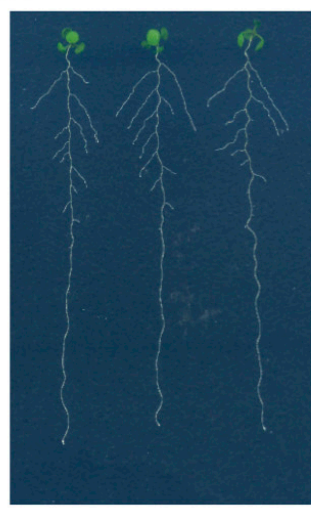

(c)

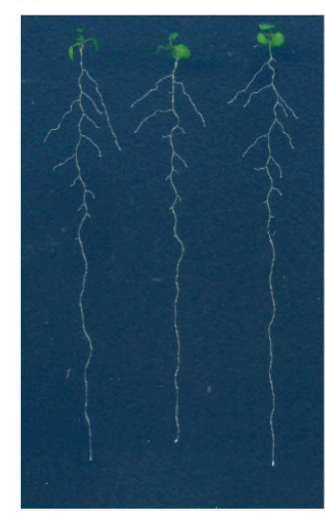

(d)

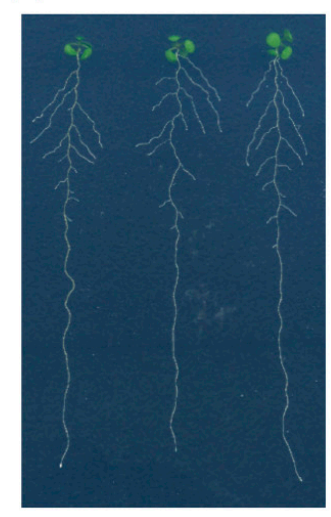

Figure 1. Effect of sulfur (S) supply on root phenotypes of Arabidopsis thaliana (A. thaliana) seedlings. The wild-type Columbia-0 (Col-0) seedlings were grown vertically on $-S(15 \mu \mathrm{M}$ sulfate) or $+\mathrm{S}(1500 \mu \mathrm{M}$ sulfate) medium for 7 days and transferred to $-\mathrm{S}$ or $+\mathrm{S}$ medium in a reciprocal manner to be grown subsequently for 3 days. The scanned images of seedlings on Day 10 are shown. The images show the phenotypes of representative seedlings transferred from (a) $-S$ to $-S$, $(\mathbf{b})-S$ to $+S$, (c) $+S$ to $+S$, and $(\mathbf{d})+S$ to $-S$, respectively.

(a)
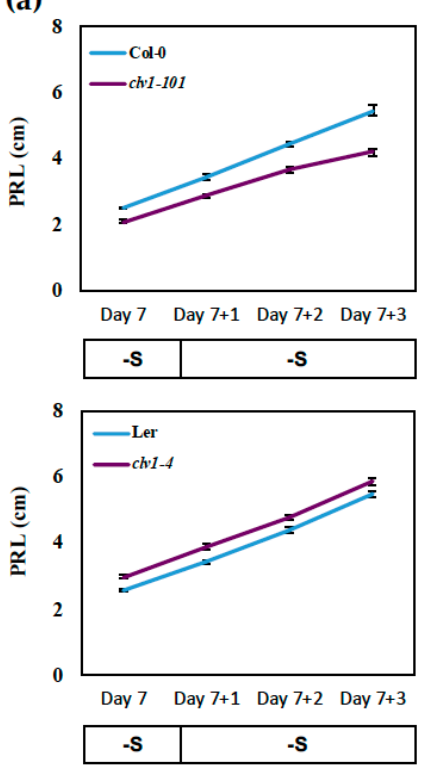

(b)
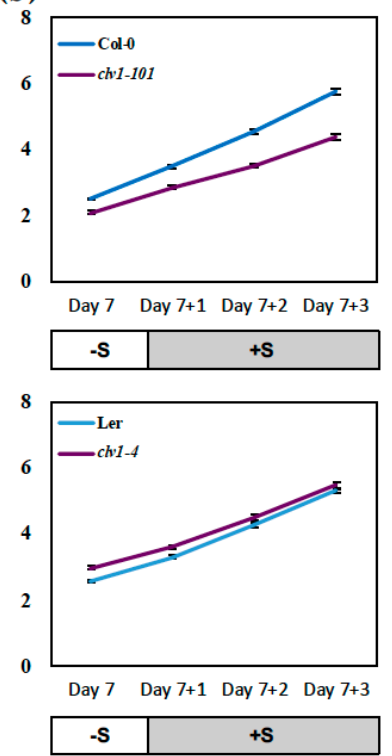

Figure 2. Effect of S supply on primary root growth. Wild-type (Col-0 and Ler) and clv1 mutant lines (clv1-101 and clv1-4) were grown vertically on $-S$ (15 $\mu \mathrm{M}$ sulfate) medium for 7 days and then transferred to (a) $-S$ medium or $(\mathbf{b})+S$ medium to be grown subsequently for 3 days. Primary root length (PRL) was measured before (Day 7) and after the transfer (Day 7+1,7+2, and 7+3) as indicated on each graph. Values show means $( \pm \mathrm{SE})$ of 24 individual plants per treatment. White and dark grey bars labeled $-S(15 \mu \mathrm{M}$ sulfate) and $+S(1500 \mu \mathrm{M}$ sulfate) below the graph represent the $S$ conditions before the transfer and during the treatment. 
As for the phenotypes associated with LR development, the LR density (LRD; total lateral root number divided by primary root length $\left(\mathrm{cm}^{-1}\right)$ ) was maintained at low levels in the wild-type Col-0 seedlings during the period of $S$ limitation which was extended for 3 days after the transfer, while it was restored upon sulfate supply and became higher during the time course (Figure 3). The LRD showed a trend of linear increase over the time course, for which the slope value can be calculated based on linear regression. The slope value of the linear regression line is expressed as the number of LR developed in one $\mathrm{cm}$ unit length of PR per day, indicating the daily increase rate of LRD. Thus, it provides a quantitative measure for the assessment of incremental changes in LR development demonstrated over time as a part of the RSA phenotype (Figure 1). The slope values were 0.36 on $+S$ and 0.20 on $-S$ medium, respectively (Figure $3 a, b$ ), suggesting that LR emergence which gave rise to visible LR happened 1.8-fold more frequently in Col-0 roots transferred to sulfate-supplied conditions compared to those exposed to prolonged $\mathrm{S}$ deficiency. These estimations were generally consistent with the visible phenotypes (Figure 1). Similar changes were observed in the wild-type Ler seedling; however, the differences between the slope values ( 0.41 on $+S$ and 0.35 on $-S$ medium, respectively) were not as significant as those estimated for Col-0 (Figure 3a,b).

(a)
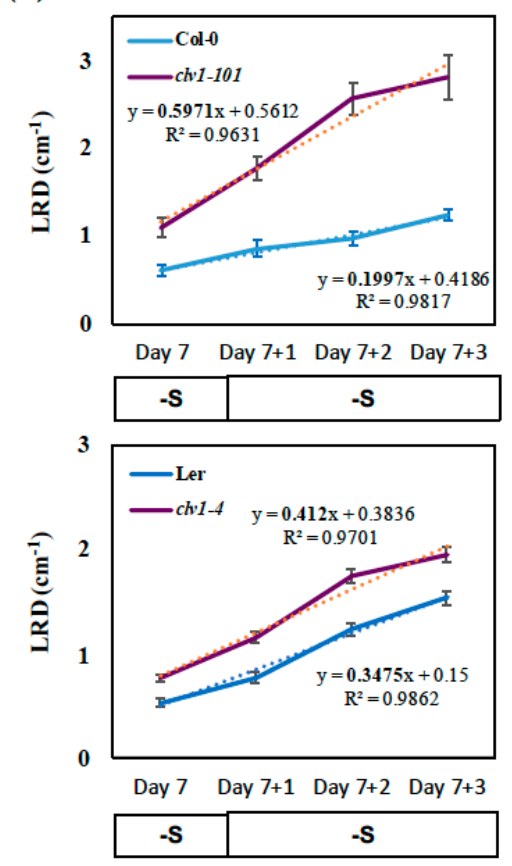

(b)
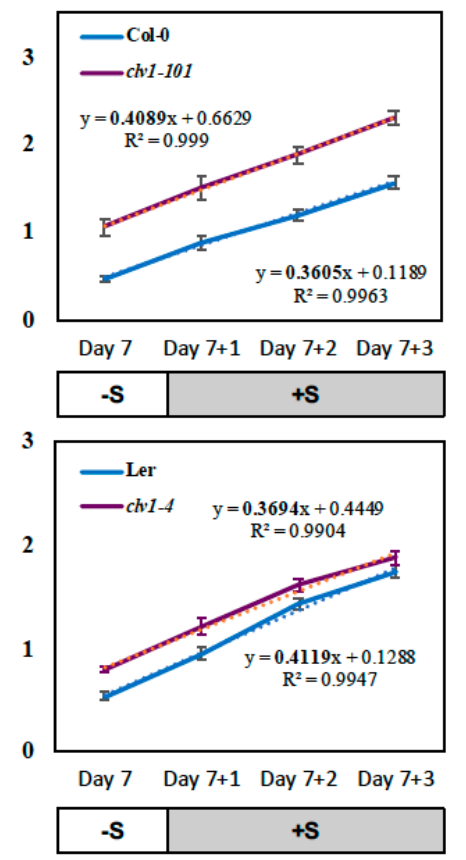

Figure 3. Effect of S supply on lateral root density (LRD). Wild-type (Col-0 and Ler) and clv1 mutant lines (clv1-101 and clv1-4) were grown vertically on $-\mathrm{S}$ (15 $\mu \mathrm{M}$ sulfate) medium for 7 days and then transferred to (a) $-S$ medium or $(\mathbf{b})+S$ medium to be grown subsequently for 3 days. LRD was calculated at each time point based on the number of emerged lateral roots (LRs) and the length of the primary root (PR) of one seedling. Values represent means ( \pm SE) of 24 individual plants per treatment. The equations for the linear repression and the R-squared values are indicated on each graph.

Consistent with our previous findings [19], LRD was constantly higher in the clv1 mutants than in the wild-type (Figure 3). LRD increased significantly in the clv1 mutants during the period of prolonged $\mathrm{S}$ deficiency (Figure 3a; transfer from $-\mathrm{S}$ to $-\mathrm{S}$ ) compared to those transferred to the sulfate-supplied medium (Figure $3 \mathrm{~b}$; transfer from $-\mathrm{S}$ to $+\mathrm{S}$ ). In contrast, the wild-type seedlings showed a lower increase rate of LRD on the $-\mathrm{S}$ medium compared to those transferred to the $+\mathrm{S}$ medium. Based on the slope values of the linear regression, the daily increase rate of LRD was estimated to be 3-fold greater in the clv1-101 mutant than in Col-0 under S-deficient conditions (Figure 3a). In contrast, upon sulfate supply, the slope value representing the increase rate of LRD was diminished in clv1-101 though 
increased in Col-0, apparently converging to the same level (Figure 3b). Similar trends were found when the clv1-4 mutant was compared with Ler, while the differences were not so obvious as those shown with the clv1-101 mutant and Col-0. These results indicated that CLV1 is a signaling component which is necessary for regulation of LR development under S-deficient conditions.

Lateral root branching density (BD) is an alternative measure of LR density, which is calculated by the number of emerged LRs divided by the length of the branching zone (the distance from shoot base to the youngest emerged LR) [29]. BD was calculated 2 and 3 days after the transfer of seedlings to $-S$ or $+S$ medium from 7 days of preculture on the $-S$ medium (Supplemental Figure S1). Consistent with the results shown for LRD (Figure 3), the wild-type plants exposed to prolonged $\mathrm{S}$ deficiency showed low BD, while this branching phenotype was recovered upon sulfate supply (Supplemental Figure S1). BD was significantly higher in the clv1 mutants compared to the wild-type under prolonged S deficiency, while it was lowered after S supplementation. These changes in BD implicate that LR primordia located between the emerged LRs are partially arrested or delayed in progression in the wild-type under the prolonged S deficiency, while their growth can be recovered by the S supplementation. These results also indicate that CLV1 is involved in the regulatory pathway that inhibits the growth of LR primordia, as shown by an increase in BD in the clv1 mutants relative to the wild-type (Supplemental Figure S1), which confirms our previous findings providing evidence for its essential role in regulating developmental stage progression of LR primordia [19]. Our present findings suggest that long-term S deficiency signals to the CLV1-directed pathway to modulate LR development as demonstrated by changes in LRD and BD.

The total LR length density (TLRLD; total lateral root length divided by primary root length $\left(\mathrm{cm} \mathrm{cm}^{-1}\right)$ ) was also calculated based on the measurement of the length of the entire LR developed in the root system (Figure 4). The clv1 mutants showed a significant increase in TLRLD during the period of prolonged $S$ limitation, where an enhanced response to $-S$ was identified in comparison with $+S$. In contrast, the wild-type showed a more significant increase in TLRLD after sulfate supplementation. These opposing effects of S limitation and sulfate supply on TLRLD in the clv1 mutants and the wild-type were consistent with those identified for LRD and BD (Figure 3 and Supplemental Figure S1). These results further suggested the essential role of CLV1 in regulation of LR development under $S$ deficiency. As shown in Figure 1 , the effect of $+S$ to $+S$ and $+S$ to $-S$ transfer on root morphology was examined simultaneously in this study. In contrast to the results obtained from the $-S$ to $-S$ and the $-S$ to $+S$ transfer experiments (Figures 3 and 4 ), the LRD and TLRLD of the $+S$-precultured seedlings changed only slightly in response to $S$ supplementation and $S$ removal (Supplemental Figures S2 and S3). The LRD and TLRLD were higher in the clv1 mutants than the wild-type as expected, but there was no substantial effect of $S$ in contrast to the phenotypes identified in roots transferred from the $-S$ preculture to $-S$ and $+S$ conditions (Figures 3 and 4 ). 
(a)
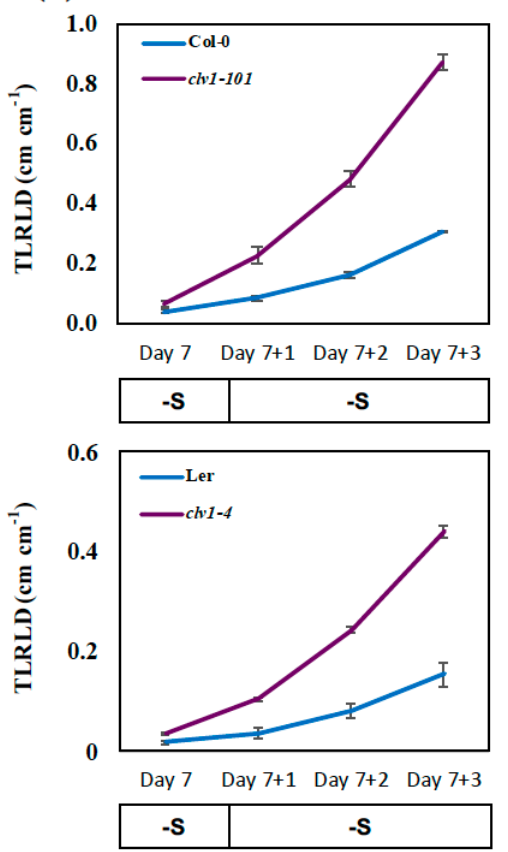

(b)
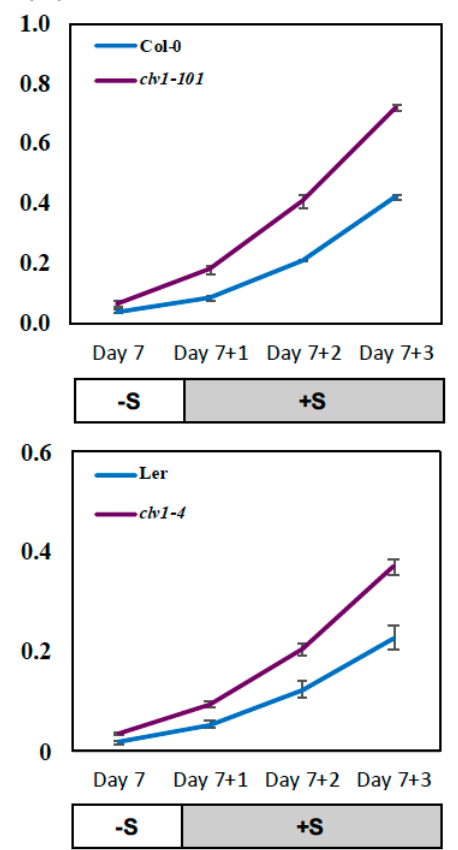

Figure 4. Effect of S supply on total lateral root length density (TLRLD). Wild-type (Col-0 and Ler) and clv1 mutant lines (clv1-101 and clv1-4) were grown vertically on $-\mathrm{S}$ (15 $\mu \mathrm{M}$ sulfate) medium for 7 days and then transferred to (a) $-S$ medium or (b) $+S$ medium to be grown subsequently for 3 days. TLRLD was calculated at each time point based on the lengths of the entire LR in one seedling and the length of the PR. Values show means $( \pm \mathrm{SE})$ of 24 individual plants per treatment.

\subsection{Regulation of CLE2 and CLE3 Gene Expression under S Deficiency}

To investigate the responses of CLE gene expression to the alteration of $S$ nutritional status, the wild-type and clv1 mutant lines were grown with different $S$ supplies as indicated above for the root phenotype analysis. The root RNAs were extracted at Day 10 (i.e., Day 7+3) for the gene expression analysis. Among the CLE genes, CLE2 and CLE3 were selected for gene expression analysis, because CLE2 was previously reported as a S-responsive CLE gene [24], and CLE3 was shown to be significantly up-regulated by $\mathrm{N}$ deficiency and inhibits LR development through acting on the CLV1 signaling pathway [19]. SULTR1;1 encodes a high-affinity sulfate transporter which is highly regulated in response to sulfur deficiency (-S) in the epidermis and cortex of $A$. thaliana roots [30,31]. Therefore, SULTR1;1 was used as an indicator for tracking the changes in $S$ status. The results indicated prolonged $S$ deficiency (transfer from $-S$ to $-S$ ) caused repression, although the sulfate replenishment (transfer from $-S$ to $+S$ ) allowed induction of CLE2 and CLE3 gene expression in both wild-type and clv1 mutant lines $(P<0.05)$ (Figure 5a,b). CLE3 showed greater responses ( $2-3$ fold) to $S$ than CLE2 $(1.2-1.5$ fold).

To investigate the effect of $S$ on CLV1-mediated feedback regulation, the $c l v 1 /$ wild-type ratios of the CLE2 and CLE3 mRNA levels were calculated and compared between the prolonged $-S$ (transfer from $-S$ to $-S$ ) and the sulfate supplied (transfer from $-S$ to $+S$ ) conditions. The results indicated both the clv1-101/Col-0 and clv1-4/Ler ratios of the CLE3 mRNA levels under the prolonged -S conditions were higher compared to those estimated for roots transferred to $+S$ medium, suggesting that prolonged $S$ deficiency activates a pathway downstream of CLV1 to feedback regulate CLE3. In contrast to CLE2 and $C L E 3$, the CLV1 expression levels did not change significantly by perturbation of $S$ supply (Figure 5c). SULTR1;1 was upregulated in roots exposed to prolonged $S$ deficiency while repressed upon sulfate replenishment, showing typical patterns of its S-responsive gene expression, which verified that the $S$ conditions tested in this study were appropriate (Figure $5 d$ ). 
(a)
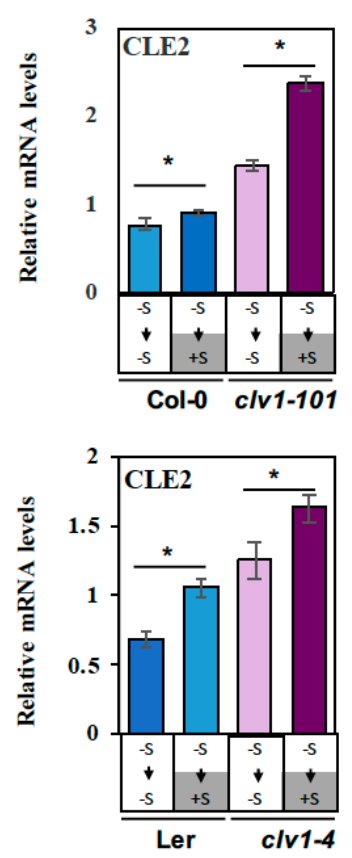

(b)
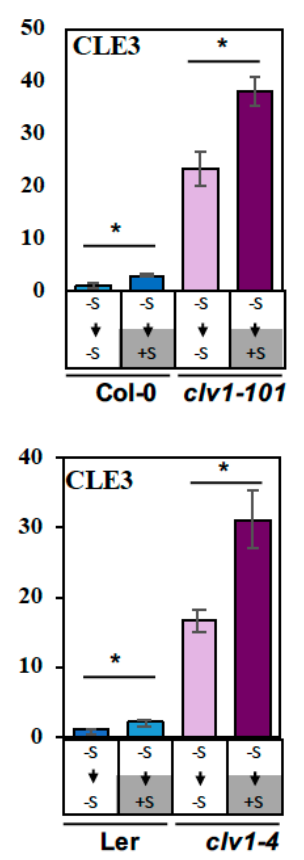

(c)
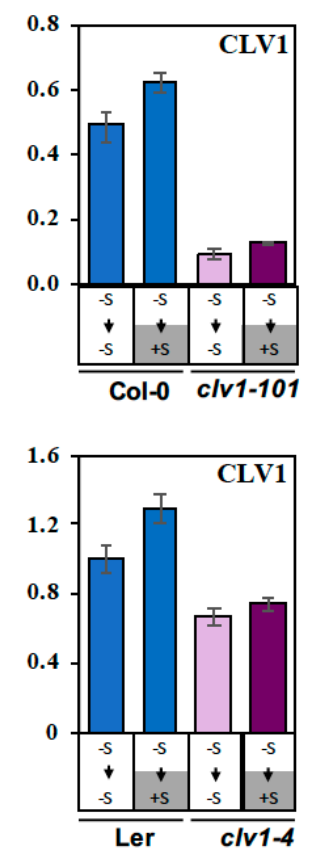

(d)
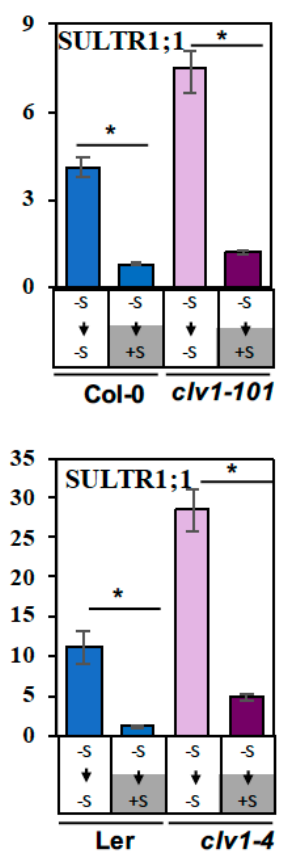

Figure 5. Regulation of CLE2 and CLE3 transcript levels by $S$ deprivation and sulfate supply. Wild-type (Col-0 and Ler) and clv1 mutant lines (clv1-101 and clv1-4) were grown vertically on $-\mathrm{S}$ (15 $\mu \mathrm{M}$ sulfate) medium for 7 days and then transferred to $-S$ medium or $+S$ medium to be grown subsequently for 3 days. The mRNA levels of (a) CLE2, (b) CLE3, (c) CLV1 and (d) SULTR1;1 in roots at Day 10 (i.e., Day 7+3) were determined by real-time PCR. Roots of wild-type plants grown on the $+S$ medium for 10 days were used as reference samples for relative quantifications. Actin 2 and Ef1 $\alpha$ were used as internal controls. Mean values $( \pm \mathrm{SE})$ of 4 biological replicates with 8 plants for each replicate were calculated using two internal controls. Asterisks indicate statistically significant differences $(P<0.05)$ between gene expression on $-S$ and $+S$ treatment. The $S$ conditions and the order of transfers are shown by white and dark grey bars and arrows below the graph.

\section{Discussion}

The results shown in this study indicate that the CLE-CLV1 signaling pathway is involved in regulation of LR development under prolonged S deficiency. The CLE-CLV1 signaling module has direct impact on LR development and physiological responses associated with changes in RSA. The proposed model describes three steps of the S-dependent signals and their coordinated actions controlling the CLE-CLV1-dependent pathway, extending our knowledge on S nutrient signaling mechanisms involved in regulation of RSA (Figure 6).

The inhibition of LR development occurs in the wild-type plants exposed to prolonged S deficiency, while this inhibition is abolished in the clv1 mutants (Figures 3 and 4). In contrast, transferring the seedlings to the S-sufficient medium leads to a recovery of LR development in the wild-type, but rather a diminished response in the clv1 mutants. The increase rate of LRD calculated based on linear regression provides additional information allowing for a quantitative interpretation of the LR phenotypes, as it is shown to be altered in response to $S$ conditions and different among the genotypes being tested in this study. As described in Figure 3, the daily increase rate of LRD was greater in the clv1 mutant than in the wild-type during the period of prolonged $S$ deficiency, but estimated to be similar between the clv1 mutant and the wild-type after the sulfate supply. These findings suggest that CLV1 is a signaling component acting on pathways negatively controlling LR development. Regulatory components expressed downstream of CLV1 may be activated under S-deficient conditions (Figure 6). The number of the emerged and visible LR was counted in experiments performed in this study. Thus, we assume that an increase in LRD after the transfer of precultured seedlings to the new medium 
corresponds to the number of newly emerged LR, and it is associated with developmental stage transition of LR primordia-as has been shown previously [19]. The inhibition of LR development under S deficiency and its CLV1 dependency may reflect these developmental mechanisms previously shown with relevance to the $\mathrm{N}$ starvation responses [19].

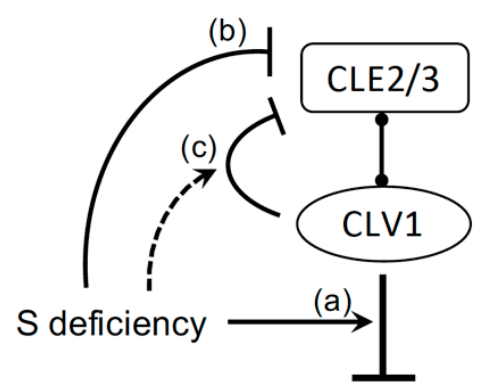

\section{Lateral Root Development}

Figure 6. Schematic model of signaling pathway controlling lateral root development under S-deficient conditions. S availability affects the activity of the signaling module composed of CLAVATA3 (CLV3)/EMBRYO SURROUNDING REGION (CLE) peptides and CLAVATA1 (CLV1) leucine-rich repeat receptor kinase in roots. The model proposes three potential pathways involved in regulation of LR development under S-deficient conditions. Regulatory components expressed downstream of CLV1 become active under S deficiency and inhibit LR development (a). CLE2 and CLE3 can be repressed under S deficiency directly or partially through the CLV1-mediated feedback mechanism (at least for CLE3) to reduce the input of CLE signals $(\mathbf{b}, \mathbf{c})$.

CLE2 and CLE3 transcript levels decrease under S deficiency in both the wild-type and the clv1 mutant lines (Figure 5). These transcript profiles suggest that CLE2 and CLE3 are repressed under S-deficient conditions in a CLV1-independent manner. In addition, changes in the clv1/wild-type ratios of the CLE3 transcript levels indicate that the CLV1-dependent feedback control mechanism may be strengthened under S-deficient conditions (Figure 5). However, the CLE2 and CLE3 transcript levels being reduced in the wild-type plants under S-deficient conditions had no positive impact on promoting LR development. As shown in our model, components expressed downstream of CLV1 are suggested to be more crucial and directly involved in regulation of LR development (Figure 6). The CLE2 and CLE3 transcript repression occurring under S-deficient conditions and partially in conjunction with the CLV1-mediated feedback pathway may be considered mechanisms that counteractively reduce the amplitude of the input CLE signals (Figure 6). CLE2 and CLE3 are expressed in the pericycle, while CLV1 is expressed in the phloem companion cells [19]. CLE2 expression is also found in the vascular tissue at the base of developing LR primordia [19,32]. It is suggested that these CLE peptides are SSPs secreted from the pericycle and diffused toward the phloem companion cells to bind to the receptor CLV1, and the CLV1-downstream components expressed in the phloem possibly carry the information to regulate development of LR primordia in long distance via trafficking through the phloem connection [19,33]. The CLE3-CLV1 ligand-receptor relationship and the potential long-distance effect of this signaling module are supported by evidence showing strong inhibition of LR development in transgenic lines with CLE3 gene overexpression driven by its own promoter in the wild type background and an apparent insensitive response to the same transgene overexpression observed in the clv1 mutant [19]. Our previous findings provide further implication that additional CLV1-downstream signals may be present and in turn sent back to the pericycle to feedback control CLE gene expression [19,33]. According to these models, both CLE peptides and CLV1-downstream components are likely transported between the two different cell types, i.e., pericycle and phloem, and through the vascular system. The actual signals involved in these putative long distance pathways yet remain to be identified. 


\section{Materials and Methods}

\subsection{Plant Growth Conditions}

Two Arabidopsis thaliana accessions, Columbia-0 (Col-0) and Landsberg erecta (Ler), and their corresponding clv1 mutants, clv1-101 and clv1-4 were used in this study. Plants were grown vertically on a nutrient medium containing 1\% agar and 1\% sucrose as described previously in Reference [19] in growth chambers (CU-36L4; Percival Scientific, Perry, IA, USA) conditioned at $22{ }^{\circ} \mathrm{C}$ under 16h-light/8h-dark long-day cycles with $75 \mu \mathrm{mol} \mathrm{m}{ }^{-2} \mathrm{~s}^{-1}$ light intensity. Agar was washed 6 times with 1 liter of deionized water and remaining water was poured off after each agar settlement to remove sulfate. S-replete $(+S)$ medium contained $1500 \mu \mathrm{M} \mathrm{MgSO}_{4}$ as the sulfur source. S-deficient (-S) medium contained $15 \mu \mathrm{M} \mathrm{MgSO}_{4}$, and $\mathrm{Mg}$ concentration was adjusted to $1500 \mu \mathrm{M}$ by adding $\mathrm{MgCl}_{2}$. After $7 \mathrm{~d}$ preculture on $+\mathrm{S}$ or $-\mathrm{S}$ medium, the seedlings were transferred to plates with the same or different concentration of sulfate and grown for $3 \mathrm{~d}$.

\subsection{Root Analysis}

Roots on agar plates were scanned at Day 7 before transfer and the following 3 days after transfer by using a scanner (Epson Perfection V700 Photo; Seiko Epson, Suwa, Japan) at 300 dpi. The images of roots were traced and measured using ImageJ. LR number, LR length, and PR length were recorded for calculation of LR density (LRD, $\mathrm{cm}^{-1}$ ) and total LR length density (TLRLD, $\mathrm{cm} \mathrm{cm}^{-1}$ ). Total LR length (TLRL, $\mathrm{cm}$ ) is the sum of the entire lateral root length per plant.

\subsection{Quantitative Real-Time PCR}

Roots were homogenized by using TissueLyser II (Qiagen, Hilden, Germany). Total RNAs were extracted by using E.Z.N.A. ${ }^{\circledR}$ Plant RNA Kit (Omega Bio-Tek, Norcross, GA, USA). Turbo DNA-free kit (Invitrogen, Thermo Fisher Scientific, Waltham, MA, USA) was used for genomic DNA removal of RNA samples. First-strand complementary DNA (cDNA) was prepared from $500 \mathrm{ng}$ of root total RNA by using SuperScript III First-Strand Synthesis System (Invitrogen, Thermo Fisher Scientific). Quantitative real-time PCR was performed by using SYBR Select Master Mix (Applied Biosystems, Thermo Fisher Scientific) on a QuantStudio 7 Flex Real-Time PCR System at the Research Technology Support Facility (RTSF) of Michigan State University. The primers were previously published $[19,31,34]$.

Supplementary Materials: The following are available online at http://www.mdpi.com/2223-7747/8/4/103/s1, Figure S1: Effect of S supply on lateral root branching density, Figure S2: Effect of S removal on lateral root density (LRD), Figure S3: Effect of S removal on total lateral root length density (TLRLD).

Author Contributions: W.D., and H.T. designed the research. W.D. performed the plant vertical culture, qRT-PCR, and data analysis. Y.W. performed the root measurements using ImageJ. W.D. wrote the paper. H.T. reviewed and edited the paper.

Funding: The authors acknowledge funding support from the National Science Foundation (MCB-1244300, IOS-1444549) and the USDA National Institute of Food and Agriculture (Hatch Project 1002395 and 1018575).

Acknowledgments: The authors thank Hiroo Fukuda (University of Tokyo) for kindly providing the clv1 mutant lines for this research.

Conflicts of Interest: The authors declare no conflict of interest. The funders had no role in the design of the study; in the collection, analyses, or interpretation of data; in the writing of the manuscript, or in the decision to publish the results.

\section{References}

1. Giehl, R.F.H.; von Wirén, N. Root nutrient foraging. Plant Physiol. 2014, 166, 509-517. [CrossRef] [PubMed]

2. Gruber, B.D.; Giehl, R.F.H.; Friedel, S.; von Wirén, N. Plasticity of the Arabidopsis root system under nutrient deficiencies. Plant Physiol. 2013, 163, 161-179. [CrossRef]

3. Lynch, J.P. Steep, cheap and deep: An ideotype to optimize water and $\mathrm{N}$ acquisition by maize root systems. Ann. Bot. 2013, 112, 347-357. [CrossRef] [PubMed] 
4. Ristova, D.; Busch, W. Natural variation of root traits: from development to nutrient uptake. Plant Physiol. 2014, 166, 518-527. [CrossRef] [PubMed]

5. Paez-Garcia, A.; Motes, C.M.; Scheible, W.-R.; Chen, R.; Blancaflor, E.B.; Monteros, M.J. Root traits and phenotyping strategies for plant improvement. Plants 2015, 4, 334-355. [CrossRef] [PubMed]

6. Gao, Y.; Tian, Q.; Zhang, W.-H. Systemic regulation of sulfur homeostasis in Medicago truncatula. Planta 2014, 239, 79-96. [CrossRef] [PubMed]

7. Gao, Y.; Li, X.; Tian, Q.-Y.; Wang, B.-L.; Zhang, W.-H. Sulfur deficiency had different effects on Medicago truncatula ecotypes A17 and R108 in terms of growth, root morphology and nutrient contents. J. Plant Nutr. 2016, 39, 301-314. [CrossRef]

8. Hubberten, H.-M.; Hesse, H.; Hoefgen, R. Lateral root growth in sulphur enriched patches. In Sulfur Metabolism in Higher Plants; Sirko, A., De Kok, L.J., Haneklaus, S., Hawkesford, M.J., Rennenberg, H., Saito, K., Schnug, E., Stulen, I., Eds.; Backhuys Publishers: Leiden, The Netherlands, 2009; pp. 105-108.

9. Dan, H.; Yang, G.; Zheng, Z.-L. A negative regulatory role for auxin in sulphate deficiency response in Arabidopsis thaliana. Plant Mol. Biol. 2007, 63, 221-235. [CrossRef]

10. Hopkins, L.; Parmar, S.; Bouranis, D.L.; Howarth, J.R.; Hawkesford, M.J. Coordinated expression of sulfate uptake and components of the sulfate assimilatory pathway in maize. Plant Biol. 2004, 6, 408-414. [CrossRef]

11. Kutz, A.; Müller, A.; Hennig, P.; Kaiser, W.M.; Piotrowski, M.; Weiler, E.W. A role for nitrilase 3 in the regulation of root morphology in sulphur-starving Arabidopsis thaliana. Plant J. 2002, 30, 95-106. [CrossRef]

12. Hell, R.; Hillebrand, H. Plant concepts for mineral acquisition and allocation. Curr. Opin. Biotechnol. 2001, 12, 161-168. [CrossRef]

13. López-Bucio, J.; Cruz-Ramírez, A.; Herrera-Estrella, L. The role of nutrient availability in regulating root architecture. Curr. Opin. Plant Biol. 2003, 6, 280-287. [CrossRef]

14. Mohd-Radzman, N.A.; Laffont, C.; Ivanovici, A.; Patel, N.; Reid, D.; Stougaard, J.; Frugier, F.; Imin, N.; Djordjevic, M.A. Different pathways act downstream of the CEP peptide receptor CRA2 to regulate lateral root and nodule development. Plant Physiol. 2016, 171, 2536-2548. [PubMed]

15. Roberts, I.; Smith, S.; Stes, E.; De Rybel, B.; Staes, A.; van de Cotte, B.; Njo, M.F.; Dedeyne, L.; Demol, H.; Lavenus, J.; et al. CEP5 and XIP1/CEPR1 regulate lateral root initiation in Arabidopsis. J. Exp. Bot. 2016, 67, 4889-4899. [CrossRef] [PubMed]

16. Imin, N.; Mohd-Radzman, N.A.; Ogilvie, H.A.; Djordjevic, M.A. The peptide-encoding CEP1 gene modulates lateral root and nodule numbers in Medicago truncatula. J. Exp. Bot. 2013, 64, 5395-5409. [CrossRef]

17. Delay, C.; Imin, N.; Djordjevic, M.A. CEP genes regulate root and shoot development in response to environmental cues and are specific to seed plants. J. Exp. Bot. 2013, 64, 5383-5394. [CrossRef]

18. Tabata, R.; Sumida, K.; Yoshii, T.; Ohyama, K.; Shinohara, H.; Matsubayashi, Y. Perception of root-derived peptides by shoot LRR-RKs mediates systemic N-demand signaling. Science 2014, 346, 343-346. [CrossRef] [PubMed]

19. Araya, T.; Miyamoto, M.; Wibowo, J.; Suzuki, A.; Kojima, S.; Tsuchiya, Y.N.; Sawa, S.; Fukuda, H.; von Wirén, N.; Takahashi, H. CLE-CLAVATA1 peptide-receptor signaling module regulates the expansion of plant root systems in a nitrogen-dependent manner. Proc. Natl. Acad. Sci. USA 2014, 111, 2029-2034. [CrossRef] [PubMed]

20. Cho, H.; Ryu, H.; Rho, S.; Hill, K.; Smith, S.; Audenaert, D.; Park, J.; Han, S.; Beeckman, T.; Bennett, M.J.; et al. A secreted peptide acts on BIN2-mediated phosphorylation of ARFs to potentiate auxin response during lateral root development. Nat. Cell Biol. 2014, 16, 66-76. [CrossRef]

21. Kumpf, R.P.; Shi, C.-L.; Larrieu, A.; Stø, I.M.; Butenko, M.A.; Péret, B.; Riiser, E.S.; Bennett, M.J.; Aalen, R.B. Floral organ abscission peptide IDA and its HAE/HSL2 receptors control cell separation during lateral root emergence. Proc. Natl. Acad. Sci. USA 2013, 110, 5235-5240. [CrossRef]

22. Shinohara, H.; Mori, A.; Yasue, N.; Sumida, K.; Matsubayashi, Y. Identification of three LRR-RKs involved in perception of root meristem growth factor in Arabidopsis. Proc. Natl. Acad. Sci. USA 2016, 113, 3897-3902. [CrossRef] [PubMed]

23. Yamaguchi, Y.L.; Ishida, T.; Sawa, S. CLE peptides and their signaling pathways in plant development. J. Exp. Bot. 2016, 67, 4813-4826. [CrossRef] [PubMed]

24. Czyzewicz, N.; Shi, C.-L.; Vu, L.D.; Van De Cotte, B.; Hodgman, C.; Butenko, M.A.; De Smet, I. Modulation of Arabidopsis and monocot root architecture by CLAVATA3/EMBRYO SURROUNDING REGION 26 peptide. J. Exp. Bot. 2015, 66, 5229-5243. [CrossRef] 
25. Patterson, K.; Cakmak, T.; Cooper, A.; Lager, I.; Rasmusson, A.G.; Escobar, M.A. Distinct signalling pathways and transcriptome response signatures differentiate ammonium- and nitrate-supplied plants. Plant Cell Environ. 2010, 33, 1486-1501. [CrossRef] [PubMed]

26. De Bang, T.C.; Lay, K.S.; Scheible, W.-R.; Takahashi, H. Small peptide signaling pathways modulating macronutrient utilization in plants. Curr. Opin. Plant Biol. 2017, 39, 31-39. [CrossRef]

27. Wang, G.; Zhang, G.; Wu, M. CLE Peptide Signaling and Crosstalk with Phytohormones and Environmental Stimuli. Front. Plant Sci. 2016, 6, 1211. [CrossRef]

28. Ohyama, K.; Shinohara, H.; Ogawa-Ohnishi, M.; Matsubayashi, Y. A glycopeptide regulating stem cell fate in Arabidopsis thaliana. Nat. Chem. Biol. 2009, 5, 578-580. [CrossRef]

29. Dubrovsky, J.G.; Forde, B.G. Quantitative analysis of lateral root development: pitfalls and how to avoid them. Plant Cell 2012, 24, 4-14. [CrossRef]

30. Maruyama-Nakashita, A.; Nakamura, Y.; Tohge, T.; Saito, K.; Takahashi, H. Arabidopsis SLIM1 is a central transcriptional regulator of plant sulfur response and metabolism. Plant Cell 2006, 18, 3235-3251. [CrossRef]

31. Maruyama-Nakashita, A.; Nakamura, Y.; Watanabe-Takahashi, A.; Yamaya, T.; Takahashi, H. Induction of SULTR1;1 sulfate transporter in Arabidopsis roots involves protein phosphorylation/dephosphorylation circuit for transcriptional regulation. Plant Cell Physiol. 2004, 45, 340-345. [CrossRef]

32. Jun, J.; Fiume, E.; Roeder, A.H.K.; Meng, L.; Sharma, V.K.; Osmont, K.S.; Baker, C.; Ha, C.M.; Meyerowitz, E.M.; Feldman, L.J.; et al. Comprehensive analysis of CLE polypeptide signaling gene expression and overexpression activity in Arabidopsis. Plant Physiol. 2010, 154, 1721-1736. [CrossRef] [PubMed]

33. Araya, T.; von Wirén, N.; Takahashi, H. CLE peptides regulate lateral root development in response to nitrogen nutritional status of plants. Plant Signal. Behav. 2014, 9, e29302. [CrossRef] [PubMed]

34. Czechowski, T.; Bari, R.P.; Stitt, M.; Scheible, W.-R.; Udvardi, M.K. Real-time RT-PCR profiling of over 1400 Arabidopsis transcription factors: unprecedented sensitivity reveals novel root- and shoot-specific genes. Plant J. 2004, 38, 366-379. [CrossRef] [PubMed]

(C) 2019 by the authors. Licensee MDPI, Basel, Switzerland. This article is an open access article distributed under the terms and conditions of the Creative Commons Attribution (CC BY) license (http://creativecommons.org/licenses/by/4.0/). 